International Journal of Child, Youth and Family Studies (2014) 5(1): 4-23

\title{
WATCHING IN CHILD AND YOUTH CARE SUICIDE INTERVENTIONS: THE POTENTIAL FOR OBSERVATION PRACTICES TO BE DISENGAGING
}

\author{
Patti Ranahan
}

\begin{abstract}
As a group of practitioners who work with adolescents, child and youth care professionals are likely to encounter suicidal adolescents in their practice. Recognizing and responding to the needs of a suicidal adolescent is challenging as professionals attempt to balance their relationship with the young person while simultaneously following customary rules of suicide intervention such as engaging in observational practices. Findings are presented from a larger constructivist grounded theory study that explains the problem of balancing physical proximity with relational proximity in observational practices with suicidal adolescents. Derived from data analysis of interviews with 19 child and youth care professionals, supervisors in youth-serving organizations, educators in schools of child and youth care, and textual analysis, I identified the observational practice of watching. Such practice involves maintaining physical proximity to the adolescent, monitoring his or her movements, and documenting observations at pre-determined intervals. While the intent of watching may be to ensure safety, findings indicate that watching may position the professional peripherally to the intervention and be enacted separate from relational engagement. As a consequence, watching is viewed as a disengaging practice. Implications for practice and avenues for future research are offered.
\end{abstract}

Keywords: suicide, observation, child and youth care practice, grounded theory

Patti Ranahan, Ph.D. is an Assistant Professor in the Department of Applied Human Sciences at Concordia University, 7141 Sherbrooke St. West, Montréal, Québec, Canada, H4B 1R6. Her research interests include youth work pedagogy and practice, mental health literacies, and youth suicide prevention practice.

E-mail: Patti.Ranahan@concordia.ca 
Intervening in situations involving potential suicide can be riddled with complexities and challenges for professionals providing care to adolescents who are contemplating ending their lives. Efforts to provide professionals with guidance and support in responding to suicidal persons in the form of best practice manuals and policies abound (see, for example, Ashworth, 2001; State of Victoria, Department of Health, 2010). Central to many such guidelines is the practice of watching or maintaining various levels of observation on the adolescent. To illustrate, the New Zealand Guidelines Group and Ministry of Health document (2003) suggests that there are various levels of observation including "within reach", "same room and in sight”, and "frequent observation" that specify intervals of observation whereby the level of observation provided is matched to the level of appraised suicide risk (p. 61). Studies on the use and effectiveness of observation of suicidal persons have been undertaken (e.g., Junker, Beeler, \& Bates, 2005), however such monitoring practices have yet to be examined in suicide interventions by child and youth care professionals with adolescents.

This paper presents findings from a larger grounded theory study that examined the mental health literacy practices realized by child and youth care professionals during their encounters with suicidal adolescents. My present discussion is focused on one of the practices I identified during data analysis: watching. The descriptions provided by participants and extant texts incorporated into the analysis consistently reflected enacting observational practices in response to the adolescent. Various terms and phrases were used to denote the practice of watching such as "having an eye on" or "getting extra eyes on”, “checks”, “monitoring”, or "putting [adolescent] on suicide watch”. As some terms for watching were organization-specific, to protect anonymity I replaced such terms with "watching".

I begin with a review of the literature pertaining to observational practices with suicidal persons. Following this, an overview of the grounded theory methods used in the study is offered. In the third section, I define and describe the practice of watching and its related conditions that I identified during analysis, and provide quotations for illustration purposes from child and youth care professional and supervisor participants, and texts that were incorporated into the analysis. Lastly, I discuss the implications of child and youth care professionals' watching practice and provide suggestions for future research.

\section{A Review of the Mechanisms of Observation in Suicide Intervention}

The present paper provides the first inquiry into child and youth care professionals' observation practices in encounters with suicidal adolescents. While there is an absence of research to draw upon in the field of child and youth care specific to suicide observation practices, the concept of watching a person who is suicidal has been taken up in the literature within allied helping professions. In this section I delineate the practice of observation as defined in the literature, outline litigation concerns, and explore the tension noted in the literature between power and collaborative engagement. Lastly, I present features evident in child and youth care literature to situate the problem under 
International Journal of Child, Youth and Family Studies (2014) 5(1): 4-23

study: child and youth care professionals balancing physical proximity with relational proximity in observational practices with suicidal adolescents.

\section{Defining the Practice of Observation}

Watching is described in the literature using a variety of terms including "constant observation" (Alland, Gallagher, \& Henderson, 2003; Fletcher, 1999; Vrale \& Steen, 2005; Wheatley, Waine, Spence, \& Hollin, 2004), “formal observation” (Manna, 2010), “special observation” (Horsfall \& Cleary, 2000), or “suicide watch” (Junker et al., 2005). The various terms refer to "high levels of observation, with some degree of removal of freedom and/or belongings, with the aim of preventing suicide” (Fletcher, 1999, p. 9). For example, in Junker and colleagues' study the researchers describe a suicide watch within a correctional setting to encompass the following mechanisms: (a) constant surveillance using closed-circuit television; (b) rounds conducted by nursing staff, every 15 minutes; (c) rounds by correctional staff, every 15 minutes; and (d) direct observation (pp. 22-23). The suicidal person's fellow inmates, who had completed an education program on observation skills, conducted direct observation.

To further illustrate and define the mechanisms of observation, three different levels of observation are recommended by CRAG, the Clinical Resource and Audit Group (2002; see Table 11). CRAG publishes clinical guidelines to develop clinical effectiveness for health and allied health care professionals within Scotland.

Table 11. Description of Progressive Levels of Observation

\begin{tabular}{|l|l|}
\hline \multicolumn{1}{|c|}{ Level of Observation } & \multicolumn{1}{c|}{ Description } \\
\hline General & $\begin{array}{l}\text { The staff on duty should have knowledge of the patients' } \\
\text { general whereabouts at all times, whether in or out of the } \\
\text { ward. }\end{array}$ \\
\hline Constant & $\begin{array}{l}\text { The staff member should be constantly aware of the precise } \\
\text { whereabouts of the patient through visual observation or } \\
\text { hearing. }\end{array}$ \\
\hline Special & $\begin{array}{l}\text { The patient should be in sight and within arm's reach of a } \\
\text { member of staff at all times and in all circumstances. }\end{array}$ \\
\hline
\end{tabular}

(CRAG, 2002, p. 2).

Despite efforts to clearly define and delineate the various levels and forms of observational practices in suicide intervention in the aforementioned articles and guidelines, Janofsky (2009) suggests that there are tensions in the literature as to the importance of observation in preventing suicide and disagreement in how observation levels are described leading to confusion and improper implementation of observation policy. As evident in the presentation of findings from the current study, child and youth care professionals also defined, interpreted, and implemented watching practice in a variety of forms. 
International Journal of Child, Youth and Family Studies (2014) 5(1): 4-23

\section{Litigation Concerns}

Customary rules of suicide intervention, rather than efficacy, underpin the practice of observation. In a review of the literature from 1996 to 2009, Manna (2010) found a significant gap in the literature providing empirical evidence of the effectiveness of the practice of observation. A barrier to research evaluating observation identified by Manna may be the legal and ethical implications of withholding a practice that may be life-saving. Despite the paucity of evidence in support of observational practices, litigation concerns are well founded. For example, a recent United States Court of Appeals ruling held a nurse and the nurse's employer partially to blame for an inmate's death by suicide for not initiating close monitoring based on the inmate's affirmative response to suicide ideation ("Suicide Risk", 2011).

\section{Power and Collaboration in the Practice of Observation}

Findings in other studies have noted that policies influence how and when professionals enact observation practices. For example, a discourse analysis of a special observation policy for nurses within an in-patient psychiatric facility illuminated the power such policies have over patients and nursing practices such that "it is unlikely that either the patient or the nurse has the actual power to resist the observation prescription, or to re-negotiate it” (Horsfall \& Cleary, 2000, p. 1295). Further, Stevenson and Cutcliffe (2006) suggest the discourse surrounding observation practices such as watching hold implicit assumptions about risk and power relations that are taken for granted.

Creating opportunities for shared decision-making regarding how watching is realized may change practice for both professionals and adolescents. For example, Kettles and Paterson (2007) conducted a small pilot study to examine the introduction of more flexible observation guidelines where nursing staff within a psychiatric setting were given more autonomy to decide a patient's level of observation, rather than only following an observation procedure directed by a physician. Initially, nurses in the study were reluctant to make decisions as to the level of observation; however, results of the study indicated a shift towards multidisciplinary team decision-making or nursing decisions regarding patients' needs for observation. Patients were on high levels of observation (e.g., special observation or constant observation) less frequently or for shorter periods of time. Kettles and Paterson suggest "staff time is better spent in engagement and therapeutic intervention than sitting in corridors watching patients from a specified distance” (p. 379). Cutcliffe and Barker (2002) suggest that observational practices may be best realized as an aspect of what I interpreted as being with (see Ranahan, 2013b) or relational engagement, rather than a technical and formal procedure of observation. That is, there is a need for professionals to balance physical proximity with relational proximity in interactions with suicidal persons. 
International Journal of Child, Youth and Family Studies (2014) 5(1): 4-23

\section{The Need to Balance Physical Proximity with Relational Proximity}

The practice of constant observation may end up creating distance between nursing professionals and patients (Alland et al., 2003), can be paternalistic, and can be an activity that interferes with the formation of therapeutic relationships (Bouic, 2005). The dynamic between observation for control and safety, and observation for therapeutic relations was identified in Vrale and Steen's (2005) content analysis examining how nurses perform constant observation of patients. Participants in the study described monitoring the patient's movements to control against self-harm, while also showing interest and care, creating hope, offering conversation about everyday life and discussing with the patient that patient's experience of constant observation. Vrale and Steen found that nurses described moving together with the patients as like a "dance” while "at the same time allowing space between the partners" thus balancing "between control and therapeutic aspects” (p. 517).

A requirement that nurses balance observation with engagement when working with persons who present a risk for violence or aggression was also recommended by Mackay, Paterson, and Cassells (2005). Using in-depth unstructured qualitative interviews, Mackay and colleagues investigated the practice of observation by nurses with potentially aggressive patients. Participants described observation in terms of procedure (e.g., a nurse implementing an institutional policy to maintain the patient within arm's reach), and role and skills (maintaining safety, intervening, de-escalation and management of aggression, assessing, communication, and therapy). That is, observation for the nurse participants in Mackay et al.’s study was comprised of more than watching: "Observations also allowed the opportunity to be with, spend time with, and help the patient - the very process of the procedure allowing 'one to one' care for those thought to be most in need of it” (p. 469). Thus observation of suicidal persons may be reconceptualized as a process of engagement to refocus professionals on the interpersonal relationship with the patient, exploring the suicidal person's experience, and attending to their needs for emotional and physical security (Cutcliffe \& Barker, 2002, p. 619).

\section{Features of Child and Youth Care Practice}

Reconceptualizing observation as engagement may be aligned with child and youth care practice perspectives. While recognizing that the field of child and youth care continues to evolve (Gharabaghi \& Krueger, 2010; Little, 2011) and final or absolute descriptions of the field should be resisted (White, 2011), features of the profession are provided here as overarching themes concerning practice with young people.

Features of child and youth care practice include the therapeutic relationship (Anglin, 1999) where the personal relationship between worker and child or youth is at the centre of practice (Fewster, 1990). Child and youth care professionals engage in "relational practice” (Bellefeuille \& Jamieson, 2008, p. 38) where "the relationship is their job” (Martin, 2002, p. 116). This relationship occurs within young people’s life 
International Journal of Child, Youth and Family Studies (2014) 5(1): 4-23

space (Anglin, 1999) where practice transpires as flexible "counselling on the go" (Garfat \& Fulcher, 2011, p. 14). Krueger (1994) posits that the worker strives to be "in-synch" with the child or youth (p. 227), while Skott-Myhre and Skott-Myhre (2011) suggest that "practice is not something we do to young people" but "we indeed do with them" (p. 46).

Thus, if child and youth care practice is relational, flexible, and collaborative, how do such professionals enact observational practices when they encounter suicidal adolescents? In the following section I present an overview of the research methods used to examine the observational practices of child and youth care professionals in their encounters with suicidal adolescents.

\section{Research Methods}

Grounded theory methods offer a rich opportunity to examine social processes, such as interactions between child and youth care professionals and suicidal adolescents. Charmaz (2000) posits that grounded theory methods offer "systematic inductive guidelines for collecting and analyzing data to build middle-range theoretical frameworks that explain the collected data” (p. 509). The theory generated through analysis may be generalizable to some extent, yet it is intimately connected to the data (Morse, 2009), and specific and meaningful to the context, situation, and participants in the study (MacDonald \& Schreiber, 2001). Grounded theory research can provide theories that are centred on resolving practical problems professionals encounter in their practice (Glaser \& Strauss, 1967), such as situations involving suicide. Further, grounded theory is suitable for inquiry into "individual process, interpersonal relations and the reciprocal effects between individuals and larger social processes” (Charmaz, 1995, pp. 28-29). Professionals' suicide intervention practices are both promoted and impeded by certain conditions (Charmaz, 2006). Conditions, then, lead to further actions, which result in various consequences (Corbin \& Strauss, 2008). That is, child and youth care professionals' mental health literacy practices are not viewed as static "but as continually changing in response to evolving conditions” (Corbin \& Strauss, 1990, p. 5). The aim of grounded theory is "to build a theoretical explanation by specifying phenomena in terms of conditions that give rise to them, how they are expressed through action/interaction, the consequences that result from them, and variations of these qualifiers" (Corbin \& Strauss, 1990, p. 9). Within the substantive theory generated during analysis, I identified several practices child and youth care professionals realized during their encounters with suicidal adolescents. In this paper, I focus on the practice of watching and the influencing conditions of policies and professionals' aroused emotional states that swayed such practice during the interactional process between professional and adolescent.

The study received ethical approval from the University of Victoria Human Research Ethics Board in May 2010. Participants included the following: 10 child and youth care professionals with not more than undergraduate qualifications (i.e., diploma or bachelor's degree) from schools of child and youth care in Western Canada and selfidentified practice experience with a suicidal adolescent; four supervisors in youthserving organizations; five educators from schools of child and youth care; and extant texts provided by participants (e.g., policy documents, practice guidelines, assessment 
International Journal of Child, Youth and Family Studies (2014) 5(1): 4-23

tools). Child and youth care professionals who participated in the study had between 4 years and 25 years practice experience in a range of settings (e.g., child welfare, residential group care, psychiatric facilities, schools, community outreach, recreation, youth justice). Semi-structured interviews were conducted with each participant and the researcher transcribed each interview and provided participants with a copy of the transcription for their review. No participants made any substantive changes to their transcription. Data collection and analysis followed universal strategies of grounded theory method which include the following:

(a) simultaneous collection and analysis of data, (b) a two-step data coding process, (c) comparative methods, (d) memo writing aimed at the construction of conceptual analyses, (e) sampling to refine the researcher's emerging theoretical ideas, and (f) integration of the theoretical framework. (Charmaz, 2000, pp. 510511)

The analysis generated the substantive theory I identified as the Balancing Proximity and Perimeter process (Ranahan, 2011). In this substantive theory, the mental health literacy practices taken up by professionals were placed on a fluid continuum with professionals shifting from one practice to another based on various influencing conditions (e.g., prior education, presence of suicide protocols, perceived role of child and youth care professionals). The theory encompassed several practices including being with (Ranahan, 2013b) and flooding the zone (Ranahan, 2013a) that have been reported elsewhere. Some practices were located within the sub-process of circling care whereby professionals were relationally engaged and connected to the young person, whereas other practices were located within the sub-process of circling defensively such that a professional's primary focus shifted to establishing a perimeter of safety to defend against the threat of suicide. As noted previously, the focus of the present discussion is specifically on the practice of watching. The following section defines watching and the conditions that influenced how child and youth care professionals realized this practice.

\section{The Practice of Watching}

Watching involved child and youth care professionals monitoring the movements of the adolescent and maintaining close physical proximity. Watching was distinct from the engaging practice of being with as the intent of the practice served an alternate purpose. Being with was a practice in which the child and youth care professional was energetically present, drawing on communication skills to listen to the adolescent, attending to the adolescent's needs, and building a relational connection (see Ranahan, 2013b). Watching, on the other hand, was a monitoring activity, often structured temporally, with the intent of supervising the adolescent's actions. Child and youth care professionals were watching for any efforts made by the adolescent to attempt to act on their suicidal ideation. That is, watching was a practice that was viewed as preventing suicide, ensuring the adolescent was physically safe and kept alive.

As the practice consistently was focused on monitoring activities and physical safety and did not constitute relational engagement, watching was interpreted during the 
International Journal of Child, Youth and Family Studies (2014) 5(1): 4-23

analysis of the data as a disengaging practice. Although professionals were in close physical proximity to the adolescent, watching was often devoid of the collaboration evidenced in being with. Further, adolescents were "put on" or "placed on" a system of monitoring and may not be informed of what watching would entail, as described by a supervisor:

I'm going to be checking in on you. We don't tell them it's every 15 minutes, obviously for the reasons that you don't want to schedule, but we do let them know that we're going to be checking on them a lot.

Supervisors identified that the timing of the "checks" when watching varied from "through the night, so 5-minute checks, and checks, that's light, breathing, watching", to "put her on suicide watch which means that the worker has to go in every 2 hours to check up on her". A child and youth care professional described how the absence of collaboration in the practice of watching was evident to the adolescent as well:

I said to [the adolescent], "Do you have a plan?" And he says, "Yes I do but you're not going to know what it is. I'm not telling you anything; I'm just going to do it. You can't watch me 24 hours. You're by yourself." "Oh Frigg! ” So yeah, I had a panic attack I have to say.

Watching was not always a realistic or effective practice with the adolescent even though child and youth care professionals may be instructed not leave the adolescent alone. However, watching often took precedence over all other activities, especially when working alone, as evidenced in the following quotation from another child and youth care professional:

And I really had to pee, [laughter] but part of the protocol is do not leave the student alone and the [colleague] was already very non-supportive and so I didn't want to ask him to watch her and I wasn't sure if I could leave [peer] in charge of her, so I missed my lunch and I missed any breaks and we sat there until about 1:30 p.m. and this was started at 10 o'clock in the morning.

Observational practices outlined in documents that were incorporated into analysis were aligned with how child and youth care professionals enacted the practice of watching. Assurances of “close supervision” and “one-on-one supervision” were emphasized in practice guidelines referenced by one child and youth care professional. Agency policies also emphasized watching as not leaving the adolescent alone. To illustrate, an agency protocol included in the analysis states, "Stay with the child/youth to ensure safety". Thus, professionals may assume that watching ensured safety.

Despite the structure of watching not lending itself to meeting a child and youth care professional's own needs around breaks, or for collaboration with the adolescent as illustrated above, when someone else was available a child and youth care professional experienced a sense of reassurance from knowing others, such as parents, would be watching: 
International Journal of Child, Youth and Family Studies (2014) 5(1): 4-23

So I just felt like I had wanted them to maybe take it a little bit more seriously and I just - yeah. That's how I felt. But I knew they were, I knew they were good parents in that they, they were aware of it, and like they were watching and like they would have an eye on him and stuff like that.

Despite the efforts to observe the adolescent, watching did not always lead to the adolescent being safe from harm. Though child and youth care professionals may have received directions to watch and instructions regarding the frequency and timing of checks on the adolescent, there appeared to be a disconnect between actually knowing the adolescent's emotional state and the practice of watching. Such a disconnect may be attributed to not knowing what to watch for, as noted by this supervisor:

When this staff came in to monitor she was watching but you know, in hindsight, not all the clues and the experience of kind of what to watch for. So the young girl decided she was going to sleep. So, she rolled over and went to sleep but in her hand she had somehow managed, you know, in the washroom, (not really quite sure how) to have a blade. So, she was rolled over and going to sleep but she was cutting when the staff was actually sitting right there. And so, not until the sheets became quite crimson red did she realize and she had gotten a good, quite a deep, deep cut.

An articulated aspect of watching was maintaining physical proximity to the adolescent so they could be observed and checked for signs of life. Professionals in some encounters went to great lengths to maintain such physical proximity and keep their eyes on the adolescent. Though the following quotation is rather lengthy, I have chosen to include this child and youth care professional's description of watching to illustrate how the practice can be enacted at all costs:

And then he boots it out the door and so my co-worker and I follow him and chase him down, like walk behind him. And as he starts walking onto oncoming traffic and we're following him and we're on radio talking to the [supervisor] that's in from previous shift, being on the phone, giving a description of what he was wearing so the police can come and get him. So we start following him and instead of him going up towards the bridge, he starts walking towards the bottom pathway, which is a good sign for us because he's not going up the bridge to jump. So he sees us that we're following him and so yeah. And he just, basically we keep following him and this is happening and it's raining too at the same time. So we're like getting soaked and we're following this kid.

The dynamic of the adolescent physically trying to move away, and the professional subsequently following them in an effort to maintain physical proximity was realized in other incidents described by participants. For example, a child and youth care professional described following an adolescent in his/her car for 2 hours. Thus, maintaining physical proximity and "eyes on" the adolescent was a central aspect of the practice of watching. 
International Journal of Child, Youth and Family Studies (2014) 5(1): 4-23

Watching also was realized during encounters with suicidal adolescents as an ownership or responsibility for all events within a particular amount of time. Underlying the watching practices of these professionals were concerns regarding the potential for harm to occur to the adolescent, and the need to protect oneself from blame. In watching, professionals essentially took responsibility for the adolescent's life and engaged in activities (e.g., documentation) as a way of proving and tracking that they did what they could to keep the adolescent alive, as illustrated by this supervisor: "Every time [adolescent] cut herself, or talked about suicide I wrote an incident report. I mean, I did massive paperwork on this girl 'cause I was very concerned that we might lose her on our watch.”

Documenting the watch was an important part of how the practice of watching was enacted by professionals. Professionals were not only concerned about their responsibility for the adolescent's life, concerns about the aftermath of an adolescent's death by suicide and the ramifications on their own well-being were also evident, as described by a supervisor:

I just, I mean, is it fear? I don't know. I keep saying, "I don't want anyone dying on my watch", but, and I haven't. And I've talked with people who have had clients complete and it's devastating because for the rest of your life you're thinking "What else could I have done?" is what people have told me and I'd probably be in that same place.

Experiencing a death by suicide could place professionals in a position of questioning their abilities and actions. Taking ownership for the watch, following the watch guidelines, ensuring watching was enacted in a structured, well-documented manner, and maintaining physical proximity, were all characteristics of child and youth care professionals' watching practice during the Balancing Proximity and Perimeter process.

Certain conditions influence the professionals' movement through the Balancing process (Charmaz, 2006) and thus which practices or tactics are employed by child and youth care professionals in their encounters with suicidal adolescents. The aim of grounded theory is "to build a theoretical explanation by specifying phenomena in terms of conditions that give rise to them, how they are expressed through action/interaction, the consequences that result from them, and variations of these qualifiers" (Corbin \& Strauss, 1990, p. 9). Thus the child and youth care professionals' practice of watching was influenced by certain conditions. In the analysis of the data I identified policies and professionals' emotional arousal as the conditions that influenced watching.

\section{Policies}

Policies refer to guidelines or protocols either prescribed by the child and youth care professionals' employer or agreed upon amongst services within a community. Policies pertaining to suicide were often made available to professionals in written form as part of a larger group of documents outlining procedures employees were to follow in 
International Journal of Child, Youth and Family Studies (2014) 5(1): 4-23

a variety of situations. Child and youth care professionals and supervisors explained policies were comprised of various steps professionals were to enact should they encounter a person who was suicidal, or a situation where a person has attempted suicide. For example, some professionals were instructed by an agency policy to enact the following steps during an encounter with a suicidal child or youth:

1. Is the child or youth's life in immediate danger?

2. Stay with the child/youth to ensure safety.

3. Arrange transport to the hospital.

4. If safe transport is not available, phone 911 for ambulance/police assistance.

5. Inform the parents/guardians.

6. Inform child/youth's therapist, social worker.

The presence of a policy outlining steps for professionals to enact during an encounter with a suicidal adolescent was a condition that influenced the Balancing process. To illustrate, step one in the above policy document is aligned with child and youth care professionals' appraising practice whereby professionals measure and assign a level of suicide risk, and step two coincides with professionals' practice of watching. Policies, then, became part of the process and influence professionals' practices in the situation.

Child and youth care professionals explained how policies could constitute either helpful or unhelpful conditions, with a consequent impact on their practice. As one child and youth care professional explained, the presence of a policy created confidence they were undertaking correct action in the encounter:

So one thing I think for me because I am a "P and $P$ " [policy and procedure] person, I value that piece because for me that gives me that sense of if anything happens, the policies and procedures are there in writing and it's clear to me of how I'm to proceed in this situation, if I come across a situation like a suicidal situation our policy and procedures state that we need to follow this way. Like you know, step by step and then follow that. And that gives me peace of mind that I'm doing my job correctly because I'm following procedure. Or at least that's a guideline for me to follow and if I didn't have that then I don't know where things would, would go, right? So, so I think that's, that's an important piece that we need to have.

The presence of a policy outlining the steps to take during encounters with suicidal adolescents provided the child and youth care professional with "peace of mind". This professional also alluded to the policy providing a predictable pathway through the encounter: "if I didn't have that then I don't know where things would, would go, right?" However, not all professionals found the presence of a policy helpful in their encounters with suicidal adolescents.

Policies may not necessarily be applicable across encounters and may provide the professional with limited information. For example, in this interview excerpt one child 
International Journal of Child, Youth and Family Studies (2014) 5(1): 4-23

and youth care professional explained the policy available from the employer was

directed only towards situations where the child or adolescent had attempted suicide:

\section{Researcher - Was there a specific policy around what you were supposed to} do if you encounter a suicidal kid?

Ask the questions.

\section{Researcher - That's what it said?}

Yeah. I remember that it was a stupid thing [laughter]. Follow the suicide protocol was all in place for if a child attempted.

\section{Researcher - Oh. Okay.}

So if you went in and they're hanging from the door, whatever, there was a specific you know, what you had to do.

\section{Researcher - But there wasn't anything to tell you -}

Not if they were sort of threatening, no.

\section{Researcher - Threatening or thinking about?}

Here's some possible questions to ask. Well, here's questions. You could ask these questions.

\section{Researcher - But then nothing to tell you what to do next?}

Well then you could phone your supervisor and get guidance that way, or if there's a mental health worker, you could phone them. If they're working with a suicide prevention worker you could phone them. So there's all these "coulds", "you could" or "you could do this" or "you could do that" or "you could do the other". But no specific, it was only if one, if there was an attempt in progress what you needed to do. Don't leave them hanging. Go call the [emergency]. That's not helpful.

Thus policies impacted child and youth care professionals' practice of watching directly. Professionals followed the steps outlined in policies regarding what questions they were to ask the adolescent and who they needed to contact and how they were to observe the adolescent.

\section{Arousal}

At the intersection of arousal and watching practice, some professionals often made decisions regarding the adolescent's suicidality based on "gut feeling" or intuition. Professionals "tuned in" to their bodies in order to recognize the physical and emotional indicators of arousal. As one child and youth care professional explained, the experience of physical and emotional arousal during the encounter may have more influence on their practice than suicide education:

And to be honest with you, I just, it's a gut, and it's just a feeling that I get. It's just an intrinsic, like. I just know: a knowing of it. I. And, of course, I've had some training in some suicidology. Like I've been to a conference and I've read up on it, but it's just really, just the experience. Just being in it, and knowing, and feeling it out. I don't know how to explain it. 
International Journal of Child, Youth and Family Studies (2014) 5(1): 4-23

\section{Researcher - Are you able to tell me more? You say "gut”. What does that feel like?}

What it feels like is a clear indication. Like it's just coming from like, where it's like you're into my intuition. And it's like this kid is serious. And it just, I think I feel like it's arousal that you get from it, that you, it's a different kind of arousal when you get this kid is just bullshitting, right? It's just a different feeling and emotion and you tap into it. Like I think when you're in tune with your body and your body knowledge and how it reacts to things then you have a clear message of what that feels like.

Several of the professionals explained they experienced a physical and emotional arousal in response to the encounter with the adolescent. For example, another child and youth care professional described engaging in the practice of watching the adolescent for several hours until someone arrived to provide relief. After the colleague took over the watching practice, the professional explained how arousal influenced his/her response to other adolescents in his/her care:

I immediately made him [colleague] sit at [the adolescent's] door. I had to disengage. It was, I was just mentally just a basket case and I went down and I think became very mother-like to the boys. Not childcare-like. It was, "You're in your rooms right now. And get in there. And get to sleep. And just I don't want to talk to you. I don't want anything. You just need to settle and I'm going to clean this mess up". And I just focused on cleaning the mess. I mean at that point I'm in their faces. They settled and then I just spent the time cleaning up. And I was like shaking.

The professional disengaged with the suicidal adolescent, was physically shaking, and mentally a "basket case". The condition of arousal influenced the professional's practices such that when the professional stopped watching, she/he moved entirely away from the adolescent, was in a heightened state of arousal, and took up a directive stance with other adolescents in the program.

Child and youth care professionals' practice of watching during their encounters with suicidal adolescents was influenced by the conditions of the presence of policy documents that directed professionals to respond by watching, and by professionals' emotionally aroused state where they drew on gut feelings or intuition to guide their watching practice. In the following section I discuss the implications for practice and future research in relation to the practice of watching.

\section{Implications for Practice and Future Research}

As noted above, child and youth care professionals' watching practice was comprised of surveillance, observation, or physically following the adolescent to maintain "eyes on". Many professionals were following a direction or implementing an agency policy when they watched the adolescent, and at times, watching became a structured, time-sensitive activity (e.g., checking on the adolescent every 15 minutes 
International Journal of Child, Youth and Family Studies (2014) 5(1): 4-23

while they are sleeping). Child and youth care professionals in my study also assumed they had little influence or power in how the practice of watching was performed. As presented above, one professional followed the observation policy above all else: "I really had to pee, [laughter] but part of the protocol is do not leave the student alone." Several implications for child and youth care practice with suicidal adolescents emerged from the findings including the need for increasing professional autonomy, adopting a political praxis, and re-envisioning relational and physical proximity as simultaneous ventures.

Increasing child and youth care professionals' autonomy and/or engagement in multidisciplinary decision-making regarding the practice of watching may be beneficial for both professionals and young people. However, such a shift would require a change in how child and youth care professionals' role is perceived, by the professionals themselves, as well as by mental health care providers. Participants in my study were not only directed by policy in realizing the practice of watching, they were also instructed to perform watching by other mental health professionals.

We can put them on special attention with the understanding that we will, we remain with them until a psychiatrist can place them on [watching]. So although [watching] means a check every 15 minutes, if I'm a supervisor and I have a large concern for maybe if you were my youth, I'd put you on [watching] but I would still keep you in my face until I could get psychiatry. What often happens is the psychiatrist, it's a matter of a telephone call, will give the verbal order for [watching] and then come in and either dismiss it or continue it. But [watching] must be directed by psychiatry.

Child and youth care professionals, as evidenced in the above quotation, did not have decision-making power or influence in the implementation of the practice of watching, nor did they critically question the practice. Child and youth care professionals were clear on who directs watching, and how the practice is realized during encounters with suicidal adolescents.

Skott-Myhre and Skott-Myhre (2011) posit that child and youth care may be viewed as political praxis whereby professionals collectively join with youth and families to "challenge the existing dominant social arrangement of society" (p. 44). That is, child and youth care practice is not only comprised of relational engagement with individual adolescents; it includes challenging oppression and working collectively towards equitable conditions for all (Newbury, 2009). Child and youth care practice, then, may be envisioned more broadly to include challenging policies and practices that marginalize young people from participating fully in the community (Stuart, 2004) and in a collaborative process in suicide intervention. Challenging policies and engaging in a political praxis would require professionals to critically examine, question, and reflect on how watching is realized in their interactions with the adolescent. Barriers to such reflexivity may be the fear of blame for an adolescent's death by suicide or the discourse of observation practice. As noted previously, participants' explained that they did not want the adolescent to die when they were responsible (e.g., "I was very concerned that 
International Journal of Child, Youth and Family Studies (2014) 5(1): 4-23

we might lose her on our watch"). Further, the discursive statements of "constant attention" or "suicide watch" discussed by child and youth care professionals serve, in part, to produce and reproduce the suicidal person as risky or dangerous, in need of being monitored within a special space (e.g., "keep you in my face”). As "language both mediates and constructs our understanding of reality” (Starks \& Trinidad, 2007, p. 1374), and discourse conveys and shapes professionals' experiences (Harvey \& Adolphs, 2012), professionals must confront and contest the dominant discourse of individual risk and watch to find new collaborative ways of being with suicidal young people.

Universal procedures for observation or watching a suicidal person evidenced in the literature and in the findings from the current study displace the expertise and decision-making of the professional who is in closest relational proximity to the person and neglects the uniqueness and individuality of the person contemplating ending their life. Child and youth care professionals in my study shifted between relational proximity and physical proximity when taking up practices such as watching. Despite being in physical proximity to the adolescent while watching, professionals were less engaged in relational proximity. Child and youth care professionals' mental health literacy practices then, within the Balancing Proximity and Perimeter process, may be best realized as concurrent and simultaneous practices; that is, relational proximity and physical proximity become intertwined in youth suicide intervention practice. While some participants in my study realized such practices concurrently, some practices such as watching were often viewed by participants as distinct, stand-alone activities. When watching is understood as a stand-alone activity, professionals are situated at the periphery, rather than in relational proximity with adolescents in their care. Such a position is in stark contrast with the focus on therapeutic relationships reported in child and youth care literature previously noted. As a detached activity, watching is a disengaged practice.

\section{Avenues for Future Research}

Mann-Feder (2011) suggests that child and youth care professionals can offer life space interventions in the form of therapeutic conversations situated in the "here and now" and "in the midst of the activities of daily living" (pp. 67-68). Further, Fewster (2001) posits that all struggles are born in relationship, and can only be resolved in the context of a relationship. Thus a child and youth care response to adolescents who are suicidal may be ideally understood as a therapeutic conversation occurring in the present moment in the context of a relationship between professional and adolescent. However, the evidence presented here of professionals' watching practice denotes a different style of engagement in many encounters such that observation was realized in a mechanistic manner, often supported by policies which outlined observation as a step to be completed by a professional and sustained in the discourse of observation (e.g., "eyes-on”).

Professionals' checks on the adolescent in 15-minute intervals lies in stark contrast to Mann-Feder's (2011) "therapeutic conversations" that occur in the "here and now" (pp. 67-68). Future research can illuminate further the dynamics between professionals' relational engagement and practices such as watching that can position professionals at a distance and are in contrast to central relational features of child and youth care practice. 
International Journal of Child, Youth and Family Studies (2014) 5(1): 4-23

Additionally, if as Manna (2010) suggests, there is a paucity of evidence supporting the effectiveness of observation practices, future research may address this gap by examining the adolescents' perspectives on how monitoring their movements impacted their suicidality. As deaths by suicide still occur when individuals are being observed (Janofsky, 2009), insight into the adolescent's perspective on child and youth care professionals' practice of watching would be beneficial for advancing knowledge of how observation practices impact suicidality.

Lastly, it is clear from the findings in the present study that power dynamics are at play in the practice of watching including the professionals' restraint in questioning how the practice was realized, and how decisions were made as to what level of observation was required. Research methods, such as discourse analysis (Potter, 2004) may further develop our understanding of such issues of power located within the discourse and practice of youth suicide intervention. As an academic discipline and profession, scholars and professionals in child and youth care have an opportunity to question and critically examine such practices in suicide intervention that may be based solely on customary rules rather than evidence-based effectiveness in ensuring adolescents' safety. As White (2012) suggests, youth suicide intervention "cannot be solved, nor contained, through an exclusive reliance on pre-determined, standardized, de-contextualized interventions” (p. 42). While watching practice may be useful and effective for individual adolescents, I suggest further examination and inquiry into observational practices in child and youth care suicide interventions are needed. 
International Journal of Child, Youth and Family Studies (2014) 5(1): 4-23

\section{References}

Alland, C., Gallagher, A., \& Henderson, J. (2003). Staying close: Creating distance? The ethics of constant observation. Mental Health Practice, 7(3), 15-16. http://dx.doi.org/10.7748/mhp2003.11.7.3.15.c1777

Anglin, J. (1999). The uniqueness of child and youth care: A personal perspective. Child \& Youth Care Forum, 28(2), 143-150.

Ashworth, J. (2001). Practice principles: A guide for mental health clinicians working with suicidal children and youth. Victoria, BC: Ministry of Children and Family Development of British Columbia. Available at http://www.mcf.gov.bc.ca/mental_health/pdf/suicid_prev_manual.pdf

Bellefeuille, G., \& Jamieson, D. (2008). Relational-centred planning: A turn toward creative potential and possibilities. In G. Bellefeuille \& F. Ricks (Eds.), Standing on the precipice: Inquiry into the creative potential of child al practice (pp. 35-72). Edmonton, AB: MacEwan Press.

Bouic, L. (2005). Focus on psvchiatric observation. Mental Health Practice, 8(8), 17-19. http://dx.doi.org/10.7748/mhp2005.05.8.8.17.c1860

Charmaz, K. (1995). Grounded theory. In J. F. Smith, R. Harre, \& L. Van Langenhove (Eds.), Rethinking methods in psychology (pp. 27-49). Thousand Oaks, CA: Sage. http://dx.doi.org/10.4135/9781446221792.n3

Charmaz, K. (2000). Grounded theory: Objectivist and constructivist methods. In N. K. Denzin \& Y. S. Lincoln (Eds.), The Sage handbook of qualitative research (2nd ed., pp. 509-535). Thousand Oaks, CA: Sage.

Charmaz, K. (2006). Constructing grounded theory: A practical guide through qualitative analysis. Thousand Oaks, CA: Sage.

Clinical Resource and Audit Group (CRAG). (2002). Engaging people: Observation of people with acute mental health problems: A good practice statement. Edinburgh, UK: The Stationery Office.

Corbin, J., \& Strauss, A. (1990). Grounded theory research: Procedures, canons, and evaluative criteria. Qualitative Sociology, 13(1), 3-21. http://dx.doi.org/10.1007/bf00988593

Corbin, J., \& Strauss, A. (2008). Basics of qualitative research (3rd ed.). Thousand Oaks, CA: Sage. http://dx.doi.org/10.4135/9781452230153

Cutcliffe, J. R., \& Barker, P. (2002). Considering the care of the suicidal client and the case for "engagement and inspiring hope" or "observations". Journal of Psychiatric and Mental Health Nursing, 9(5), 611-621. 
International Journal of Child, Youth and Family Studies (2014) 5(1): 4-23

Fewster, G. (1990). Growing together: The personal relationship in child and youth care. In J. P. Anglin, C. J. Denholm, R. V. Ferguson, \& A. R. Pence (Eds.), Perspectives in professional child and youth care (pp. 25-40). Binghamton, NY: Haworth Press. http://dx.doi.org/10.1300/j024v13n01_04

Fewster, G. (2001). Going there from being here. CYC Online, 25. Retrieved from http://www.cyc- net.org/cyc-online/cycol-0201-fewster.html

Fletcher, R. F. (1999). The process of constant observation: Perspectives of staff and suicidal patients. Journal of Psychiatric and Mental Health Nursing, 6, 9-14.

Garfat, T., \& Fulcher, L. (2011). Characteristics of a child and youth care approach. Relational Child \& Youth Care Practice, 24(1/2), 7-19.

Gharabaghi, K., \& Krueger, M. (2010). A new politic in child and youth care. Relational Child \& Youth Care Practice, 23(3), 27-39.

Glaser, B. G., \& Strauss, A. L. (1967). The discovery of grounded theory: Strategies for qualitative research. Piscataway, NJ: Aldine.

Harvey, K., \& Adolphs, S. (2012). Discourse and healthcare. In J. P. Gee \& M. Handford (Eds.), Routledge handbook of discourse analysis (pp. 470-481). New York: Routledge.

Horsfall, J., \& Cleary, M. (2000). Discourse analysis of an “observation levels” nursing policy. Journal of Advanced Nursing, 32(5), 1291-1297. http://dx.doi.org/10.1046/j.1365-2648.2000.01601.x

Janofsky, J. S. (2009). Reducing inpatient suicide risk: Using human factors analysis to improve observation practices. Journal of the American Academy of Psychiatry and the Law Online, 37(1), 15-24.

Junker, G., Beeler, A., \& Bates, J. (2005). Using trained inmate observers for suicide watch in a federal correctional setting: A win-win solution. Psychological Services, 2(1), 20-27. doi: 10.1037/1541-1559.2.1.20 http://dx.doi.org/10.1037/1541-1559.2.1.20

Kettles, A. M., \& Paterson, K. (2007). Flexible observation: Guidelines versus reality. Journal of Psychiatric and Mental Health Nursing, 14(4), 373-381. http://dx.doi.org/10.1111/j.1365-2850.2007.01092.x

Krueger, M. (1994). Framing child and youth care in moments of rhythm, presence, meaning, and atmosphere. Child \& Youth Care Forum, 23(4), 223-229. http://dx.doi.org/10.1007/bf02209086

Little, N. (2011). Articulating a child and youth care philosophy: Beyond binary constructs. In A. Pence \& J. White (Eds.), Child and youth care: Critical perspectives on pedagogy, practice, and policy (pp. 3-18). Vancouver, BC: UBC Press. 
International Journal of Child, Youth and Family Studies (2014) 5(1): 4-23

MacDonald, M., \& Schreiber, R. S. (2001). Constructing and deconstructing: Grounded theory in a postmodern world. In R. S. Schreiber \& P. N. Stern (Eds.), Using grounded theory in nursing (pp. 35-53). New York: Springer.

Mackay, I., Paterson, B., \& Cassells, C. (2005). Constant or special observations of inpatients presenting a risk of aggression or violence: Nurses' perceptions of the rules of engagement. Journal of Psychiatric and Mental Health Nursing, 12(4), 464-471. http://dx.doi.org/10.1111/j.1365-2850.2005.00867.x

Manna, M. (2010). Effectiveness of formal observation in inpatient psychiatry in preventing adverse outcomes: The state of science. Journal of Psychiatric and Mental Health Nursing, 17(3), 268-273. doi: 10.1111/j.1365-2850.2009.01512.x http://dx.doi.org/10.1111/j.1365-2850.2009.01512.x

Mann-Feder, V. R. (2011). Child and youth care work and talk therapy. Relational Child \& Youth Care Practice, 24(1/2), 67-71.

Martin, L. (2002). The invisible table: Perspectives on youth and youthwork in New Zealand. Auckland, New Zealand: Dunmore Press.

Morse, J. M. (2009). Tussles, tensions, and resolutions. In J. M. Morse (Ed.), Developing grounded theory: The second generation (pp. 13-22). Walnut Creek, CA: Left Coast Press.

Newbury, J. (2009). Contextualizing child and youth care: Striving for socially just practice. Relational Child \& Youth Care Practice, 22(4), 20-29.

New Zealand Guidelines Group \& Ministry of Health. (2003). Best practice evidencebased guideline: The assessment and management of people at risk of suicide for emergency departments and mental health service acute assessment settings. Retrieved from www.nzgg.org.nz/resources/41/Suicide_Guideline.pdf

Potter, J. (2004). Discourse analysis. In M. Hardy \& A. Bryman (Eds.), Handbook of data analysis (pp. 607-624). Thousand Oaks, CA: Sage. http://dx.doi.org/10.4135/9781848608184.n27

Ranahan, P. (2011). Child and youth care professionals' mental health literacy practices in their encounters with suicidal adolescents: A grounded theory study. Doctoral dissertation, University of Victoria, Victoria, BC. Retrieved from http://dspace.library.uvic.ca:8080/handle/1828/3690?show=full

Ranahan, P. (2013a). Why did you call for them? Child and youth care professionals' practice of flooding the zone during encounters with suicidal adolescents. Child Care in Practice, 19(2), 138-161. doi: 10.1080/13575279.2012.750598 http://dx.doi.org/10.1080/13575279.2012.750598

Ranahan, P. (2013b). Being with: Child and youth care professionals' practice with suicidal adolescents. Relational Child \& Youth Care Practice, 26(1), 6-17. 
International Journal of Child, Youth and Family Studies (2014) 5(1): 4-23

Skott-Myhre, K., \& Skott-Myhre, H. A. (2011). Theorizing and applying child and youth care praxis as politics of care. Relational Child \& Youth Care Practice, 24(1/2), 42-52.

State of Victoria, Department of Health. (2010). Working with the suicidal person: Clinical practice guidelines for emergency departments and mental health services. Melbourne, Australia: Author. Retrieved from http://www.health.vic.gov.au/mentalhealth/suicide/suicidal-person-book2010.pdf

Starks, H., \& Trinidad, S. B. (2007). Choose your method: A comparison of phenomenology, discourse analysis, and grounded theory. Qualitative Health Research, 17(10), 1372-1380. doi: 10.1177/1049732307307031 http://dx.doi.org/10.1177/1049732307307031

Stevenson, C., \& Cutcliffe, J. (2006). Problematizing special observation in psychiatry: Foucault, archaeology, genealogy, discourse and power/knowledge. Journal of Psychiatric and Mental Health Nursing, 13(6), 713-721. http://dx.doi.org/10.1111/j.1365-2850.2006.01023.x

Stuart, G. (2004). Nonviolence as a framework for youth work practice. Youth Studies Australia, 23(3), 26-32.

Suicide risk: Nurse failed to act based upon patient's screening. (2011, May). Legal Eagle Eye Newsletter for the Nursing Profession, 19(5), 3.

Vrale, G. B., \& Steen, E. (2005). The dynamics between structure and flexibility in constant observation of psychiatric inpatients with suicidal ideation. Journal of Psychiatric and Mental Health Nursing, 12(5), 513-518. http://dx.doi.org/10.1111/j.1365-2850.2005.00854.x

Wheatley, M., Waine, J., Spence, K., \& Hollin, C. R. (2004). Characteristics of 80 adolescents referred for secure inpatient care. Clinical Psychology \& Psychotherapy, 11(2), 83-89. doi: 10.1002/cpp.398 http://dx.doi.org/10.1002/cpp.398

White, J. (2012). Youth suicide as a "wild” problem: Implications for prevention practice. Suicidology Online, 3, 42-50. Retrieved from http://www.suicidologyonline.com/pdf/SOL-2012-3-42-50.pdf

White, J. (2011). Re-stor(y)ing professional ethics in child and youth care: Toward more contextualized, reflexive, and generative practices. In A. Pence \& J. White (Eds.), Child and youth care: Critical perspectives on pedagogy, practice, and policy (pp. 33-51). Vancouver, BC: UBC Press. 